\title{
Design and Application of Compact and Highly Tolerant Polarization-Independent Waveguides
}

\author{
Kerstin Wörhoff, Chris G. H. Roeloffzen, Member, IEEE, René M. de Ridder, Member, IEEE, \\ Alfred Driessen, Senior Member, IEEE, and Paul V. Lambeck
}

\begin{abstract}
In this paper, the design, fabrication, and application of a highly tolerant polarization-independent optical-waveguide structure suited for operation in the third communication window is presented. The waveguide structure has been optimized toward minimized sensitivity to technological tolerances and low fabrication complexity. The tolerance analysis has been based on the typical processing tolerances of the widely applied siliconoxynitride technology, being $\pm 3 \times 10^{-4}$ in refractive index, $\pm 1 \%$ in thickness, and $\pm 0.1 \mu \mathrm{m}$ in channel width. The optimized waveguide design fulfills the criterion of a channel birefringence within $5 \times 10^{-5}$, including processing tolerance. It also enables a fiber-to-chip coupling loss below $1 \mathrm{~dB} /$ facet and is suited for the realization of low-loss bends with a radius down to $600 \mu \mathrm{m}$. Based on this waveguide design, a passband-flattened optical wavelength filter with 50-GHz free spectral range has been realized and tested. The measured TE-TM shift of $0.03 \mathrm{~nm}$ confirms the polarization dependence of the optical waveguides being as low as $3 \times 10^{-5}$.
\end{abstract}

Index Terms-Adiabatic bend, optical filter, polarizationindependent waveguide (PIW), silicon oxynitride, tolerance analysis.

\section{INTRODUCTION}

$\mathbf{T}$ HE DEVELOPMENT of a broad range of integrated optical (IO) devices for the telecommunications field has been extremely speeded up over the last decades [1], [2]. The need for such devices is mainly motivated by the strongly increased data-transfer rate required by today's worldwide communication society. As this evolution will continue in the future, it is evident that the capacity of today's communication networks will be by far not sufficient to satisfy this need. The solution to this capacity problem will be found in the introduction of all-optical networks based on IO devices [3]-[5]. For the commercialization of such devices, several general requirements have to be fulfilled: low insertion loss, polarization-independent operation, high integration density, and the accessibility of reliable low-cost fabrication technologies.

Manuscript received June 7, 2006; revised August 4, 2006. This work was supported by the Dutch Science Foundation (STW), especially for the research on the WDM.

K. Wörhoff, R. M. de Ridder, A. Driessen, and P. V. Lambeck are with the Integrated Optical MicroSystems Group, MESA+ Institute for Nanotechnology, University of Twente, 7500 AE Enschede, The Netherlands (e-mail: K.Worhoff@ewi.utwente.nl).

C. G. H. Roeloffzen was with the Integrated Optical MicroSystems Group, MESA+ Institute for Nanotechnology, University of Twente, $7500 \mathrm{AE}$ Enschede, The Netherlands. He is now with the Telecommunication Engineering Group, University of Twente, 7500 AE Enschede, The Netherlands.

Color versions of one or more of the figures in this paper are available online at http://ieeexplore.iee.org.

Digital Object Identifier 10.1109/JLT.2007.893041
In order to reach this goal, numerous concepts [1], [6]-[8], based on different materials systems and technologies, have been developed by various research groups. They range from low-contrast processes (doped silica [5], polymers [9], and doped $\mathrm{LiNbO}_{3}$ [10]) via moderate/high-contrast technologies ( $\mathrm{SiON}$ [11], [12], $\mathrm{Si}_{3} \mathrm{~N}_{4}$ [14], and $\mathrm{TiO}_{2}$ [15]) up to very highcontrast waveguides ( $\mathrm{Si}$ [16], [17] and III-V based [1], [8]). In general, it is rather difficult, if not impossible, to satisfy completely all of the above given demands on IO devices with any of the materials systems simultaneously, because several of the demands result in contrary requirements on specific waveguide properties. For example, a good fiber-to-chip coupling efficiency is easily realized with low-index-contrast waveguides, while the demand on high integration density requires large index contrasts.

The realization of polarization-independent devices is of interest due to the generally unknown polarization state of the optical data transported through the fiber network. Various concepts for compensating the mostly stress-induced materials birefringence have been reported. Compensation techniques based on different mechanisms such as stress reduction (stressreleasing grooves and thick silicon layer on top of the structure [18], [19]), adaptation of form birefringence (waveguide geometry [8], [20] and $\mathrm{Si}_{3} \mathrm{~N}_{4}$ patch below the core [21], [22]), and compensating devices (polarization converters [23] and polarization diversity) have been applied. Except for compensation by directly adapting the waveguide geometry, all other techniques will either require additional processing steps or will increase the device complexity.

The research described in this paper has focused on the design and application of compact and highly tolerant polarization-independent waveguides (PIWs), which can be fabricated with low technological complexity. The design offers a good tradeoff in fulfilling all the demands arising from the telecom-application field. Realization in plasma-enhanced chemical-vapor-deposition (PECVD) SiON technology, which becomes increasingly attractive since very recent research has shown the successful elimination of the loss-inducing hydrogen overtones in as-deposited films [24]-[26], ensures, moreover, low-cost-production feasibility. The application of our waveguide design is demonstrated by a passband-flattened optical wavelength filter.

\section{Design}

\section{A. Optical Waveguide Design}

The waveguide design is based on a simple three-layer stack, schematically depicted in Fig. 1, with a buffer and cladding 


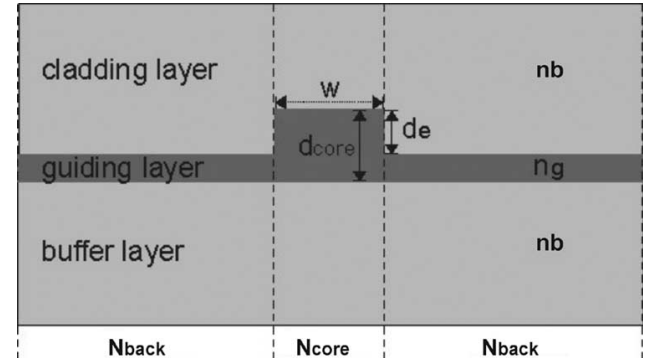

Fig. 1. Schematic layout of the waveguiding structure with an indication of all relevant parameters.

TABLE I

Summary of PARAMETER RANGE FOR THE WAVEgUIDE DESIGN (CONTRAST $\Delta n$ IS DEFINED AS $n_{g}-n_{b}$ )

\begin{tabular}{|c|c|c|c|c|}
\hline Parameter & $\Delta n_{T M-T E} \times 10^{-3}$ & contrast $\Delta n$ & $w_{\text {core }}[\mu \mathrm{m}]$ & $d_{\text {core }}[\mu \mathrm{m}]$ \\
\hline Range & $0.5-2.5$ & $0.04-0.14$ & $3-12$ & $0.3-1.5$ \\
\hline
\end{tabular}

layer having identical refractive indexes $n_{b}$ and a guiding layer with refractive index $n_{g}$ and thickness $d_{\text {core. In the }}$ guiding layer, a ridge-type waveguide channel is defined with width $w_{\text {core }}$ and ridge height $d_{e}$. The layer stack at the channel position is defined by the effective index $\left(N_{\text {core }}\right)$, while the effective index of the layer stack aside the channel, which is the so-called background, is denoted by $N_{\text {back. }}$. The effective lateral-refractive-index contrast is then given by $\Delta N=N_{\text {core }}-N_{\text {back }}$. The material birefringence, which is in our materials stress-induced, is defined by $\Delta N_{\mathrm{TM}-\mathrm{TE}}=$ $N_{\mathrm{TM}}-N_{\mathrm{TE}}$, where $N_{\mathrm{TM}}$ and $N_{\mathrm{TE}}$ are the refractive-index components, which are relevant for TM and TE polarization, respectively. Although the presented waveguide design is generic and can be applied in any waveguide technology which enables the fabrication of optical thin films with properties as described in Table I, this paper will focus on the application of SiON technology due to its availability in our laboratory [27]. In $\mathrm{SiON}$ technology, the core layer is typically sandwiched by two silicon dioxide $\left(\mathrm{SiO}_{2}\right)$ layers. Dependent on the exact deposition technique, the refractive index of silicon oxide can vary between 1.449 (thermal oxide) and 1.463 (PECVD oxide) at 1550 -nm wavelength. The material birefringence of the $\mathrm{SiO}_{2}$ layers is measured to be about $1 \times 10^{-3}$. In our approach, the birefringence properties of the guiding layer will be based on values as typically obtained for these types of structures in PECVD SiON technology, i.e., in the range of $0.5-2.5 \times 10^{-3}$ [12]. The technological tolerances of the waveguide parameters in our SiON technology have been determined to be $\Delta n_{g}=$ $\pm 3 \times 10^{-4}, \delta d= \pm 1 \%, \Delta w= \pm 0.1 \mu \mathrm{m}$, and $\Delta N_{\mathrm{TM}-\mathrm{TE}}=$ $\pm 1 \times 10^{-4}$ [12]. With respect to the technological tolerance of the last parameter (the material birefringence), it should be mentioned that the measurement error of this value was in the same order of magnitude. Therefore, it can be applied later on in the tolerance analysis for indicative purposes only.

The requirements to our waveguide technology arise, on the one hand, heuristically from considerations that losses should be sufficiently low for not interfering with device functionality and bending radius small enough for allowing the design of, e.g., ring resonators with $>0.2$-nm free spectral range (FSR). On the other hand, requirements for polarization dependence can be inferred from specifications like Telcordia GR1209. These considerations led to the following set of requirements [13]:

1) application in the wavelength range $\sim 1530-1600 \mathrm{~nm}$;

2) monomode waveguide operation;

3) bending radius $\left(R_{b}<1 \mathrm{~mm}\right)$ at bending loss $\left(\alpha_{b}<\right.$ $\left.0.01 \mathrm{~dB} / 90^{\circ}\right)$

4) polarization dependence of channel $\left(\Delta N_{\mathrm{TM}-\mathrm{TE}}<5 \times\right.$ $\left.10^{-5}\right)$

5) fiber-to-chip coupling loss $\left(\alpha_{\mathrm{fc}}<1 \mathrm{~dB} /\right.$ facet$)$ and standard single-mode fiber with $9-\mu \mathrm{m}$ core diameter;

6) waveguide loss $(\alpha<0.2 \mathrm{~dB} / \mathrm{cm})$.

In addition to these general requirements relating to performance, the technology for waveguide fabrication should be as simple and reliable as possible. This implies the choice of waveguide parameters yielding a large tolerance of its relevant properties toward the uncertainties of the fabrication process. For example, in the case of the polarization dependence, this means that the criterion of $\Delta N_{\mathrm{TM}-\mathrm{TE}}<5 \times 10^{-5}$ has to be met, taking into account the impact of all technological tolerances.

The parameter window, within which the design has been optimized, is given in Table I. This window is based on the following limitations or choices. First of all, the thickness of the guiding layer next to the channel is chosen to be zero $\left(d_{\text {core }}=\right.$ $d_{e}$ ) in order to eliminate any uncertainty in the ridge height. In that case, effects of any overetch of the core layer can be eliminated, since our technology enables fabrication of identical refractive indexes of the buffer and cladding layer. Although an as-deposited $\mathrm{PECVD} \mathrm{SiO}_{2}$ layer has a higher refractive index than thermal oxide, it can be tuned to that lower refractiveindex value by an appropriate annealing step. The choice of the contrast range is based on some preliminary calculations, which have shown that a moderate contrast is needed in order to fulfill the contradicting requirements of low fiber-to-chip coupling loss and small bending radius while simultaneously obeying the condition of low fabrication complexity. The latter condition implies that we aim at a single-mask waveguidechannel definition offering, however, the freedom of varying the channel width at the fiber-chip-coupling section [12] and bend positions [29]. Finally, we have chosen a maximum thickness of the guiding layer, $d_{\text {core }}=1.5 \mu \mathrm{m}$, and a lower limit for the channel width being $w_{\text {core }}=3 \mu \mathrm{m}$. This choice is motivated by reasons of economical and technological in nature, such as a short deposition and etching time of the core layer, small bending of the wafer due to stresses in the layer stack, enabling better lithographic resolution, reliability and reproducibility of channel fabrication, thin resist layer with a thickness just sufficient for etching the required ridge height, etc.

Within that parameter range, a large number of PIWs, all fulfilling the $\left|\Delta N_{\mathrm{TE}-\mathrm{TM}}\right|<5 \times 10^{-5}$ criterion, have been calculated, applying a finite-difference multigrid-mode-solving method [28]. At a given core refractive index and material birefringence, for each value of $d_{\text {core }}$, polarization-independent operation is obtained for a single value of $w_{\text {core }}$ only.

For some selected PIWs, all having a residual birefringence smaller than $10^{-6}$ and a core-layer refractive index of 


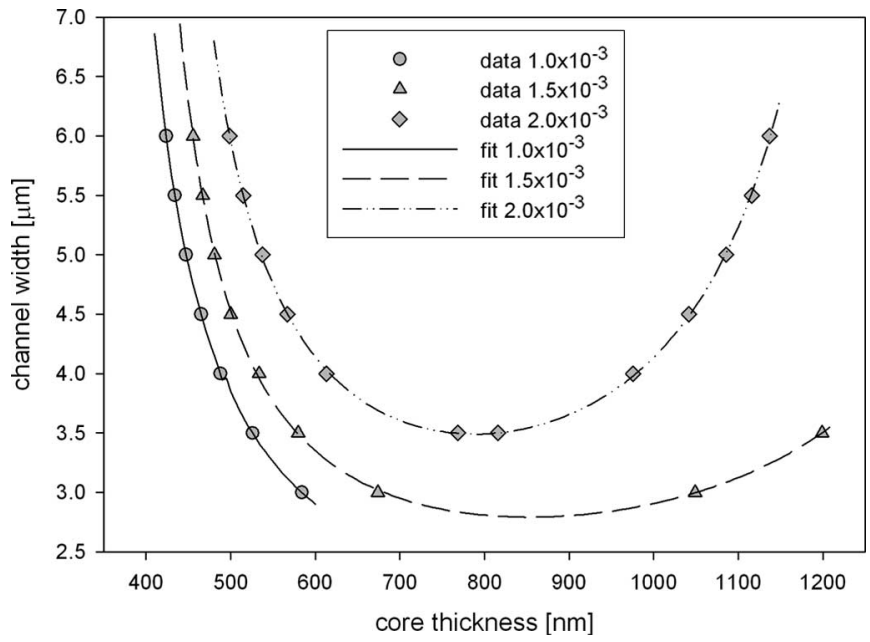

Fig. 2. Relation between core thickness and channel width for calculated PIW data points based on a 1.53 core refractive index and various material birefringence values. The lines show the fitting of the data points based on (1).

TABLE II

Summary of the Range of VARIous Restrictions on the SeT of PIW SOLUTIONS

\begin{tabular}{|c|c|c|c|}
\hline \multirow{2}{*}{$\begin{array}{l}\text { range selection } \\
\text { based on }\end{array}$} & \multicolumn{3}{|c|}{ suited parameter range } \\
\hline & $n_{g}$ & $d_{\text {core }}[\mathrm{nm}]$ & $w_{\text {core }}[\mu \mathrm{m}]$ \\
\hline monomodality & $1.51-1.60$ & $200-1200^{*}$ & $3-5.5^{*}$ \\
\hline compact bend criterion & $1.51-1.54$ & $600-1100$ & $3-3.5^{* *}$ \\
\hline technology tolerance & $1.53-1.55$ & $200-900$ & $3-6$ \\
\hline
\end{tabular}

1.53, the dependence between core thickness $d_{\text {core }}$ and channel width $w_{\text {core }}$ at three material-birefringence values $\left(1.0,1.5\right.$, and $\left.2.0 \times 10^{-3}\right)$ is shown in Fig. 2. It turned out that the data points fit excellently to a rational (1), going up to second-order terms:

$$
w=\left(a_{1}+a_{2} d+a_{3} d^{2}\right) /\left(1+a_{4} d+a_{5} d^{2}\right) .
$$

The analytical expressions allow for a fast calculation of the channel width required for a PIW at any given layer thickness, which show a residual channel birefringence of less than $10^{-6}$.

From the assembly of waveguides obeying the given polarization-dependence criterion, we select now the ones which fulfill all other requirements. First of all, solutions which are either multimodal or do not have the potential of fulfilling the compact-bend criterion have been skipped. As indicated in Table II, for each value of the core-layer index, the waveguides are monomodal for a restricted number of combinations of the values of core thickness and channel width. After the exclusion of multimodal PIWs, waveguides with potentially compact bends were selected. This selection was based on the application of the whispering-gallery-mode (WGM) model [29], and as a result, it could be concluded that the slab effective index contrast between channel and background sections $\Delta N$ had to be at least $1.9 \times 10^{-2}$. The range in which this criterion is fulfilled is also indicated in Table II. It has to be mentioned that in this table, the channel width refers to the width of the incoming straight channel. It is well known that the channel in the bend has to be broadened in order to operate
TABLE III

TOLERANCE-ANALYSIS RESUlTS FOR MAXIMUM ABSOLUTE IMPACT ON CHANNEL BIREFRINGENCE AND OBSERVED TRENDS FOR THE TECHNOLOGICAL UNCERTAINTY OF EACH WAVEGUIDE PARAMETER With APPliEd VALUes GiVEN IN BRACKETS

\begin{tabular}{|c|c|c|}
\hline Parameter & Impact & Trend \\
\hline $\begin{array}{c}\Delta n_{g} \\
\left(3 \times 10^{-4}\right)\end{array}$ & $1.5 \times 10^{-5}$ & $\begin{array}{c}\text { Decreasing at higher } n_{g} \text { and lower } \\
w_{\text {core }} ; \text { maximum for } d_{\text {core }} \sim 550 \mathrm{~nm}\end{array}$ \\
\hline $\begin{array}{c}\delta d \\
(1 \%)\end{array}$ & $2.9 \times 10^{-5}$ & $\begin{array}{c}\text { Decreasing at lower } n_{g} \text { and higher } \\
d_{\text {core }} ; \text { effect of } w_{\text {core }} \text { negligible }\end{array}$ \\
\hline $\begin{array}{c}\Delta w \\
(0.1 \mu \mathrm{m})\end{array}$ & $3.8 \times 10^{-5}$ & $\begin{array}{c}\text { Decreasing at higher } w_{\text {core }} \text { and lower } \\
n_{g} ; \text { effect of } d_{\text {core }} \text { negligible }\end{array}$ \\
\hline $\begin{array}{c}\Delta n_{T M-T E} \\
\left(1 \times 10^{-4}\right)\end{array}$ & $5.7 \times 10^{-5}$ & $\begin{array}{c}\text { Decreasing at lower } d_{\text {core }} \text { and higher } \\
n_{g} ; \text { effect of } w_{\text {core }} \text { negligible }\end{array}$ \\
\hline
\end{tabular}

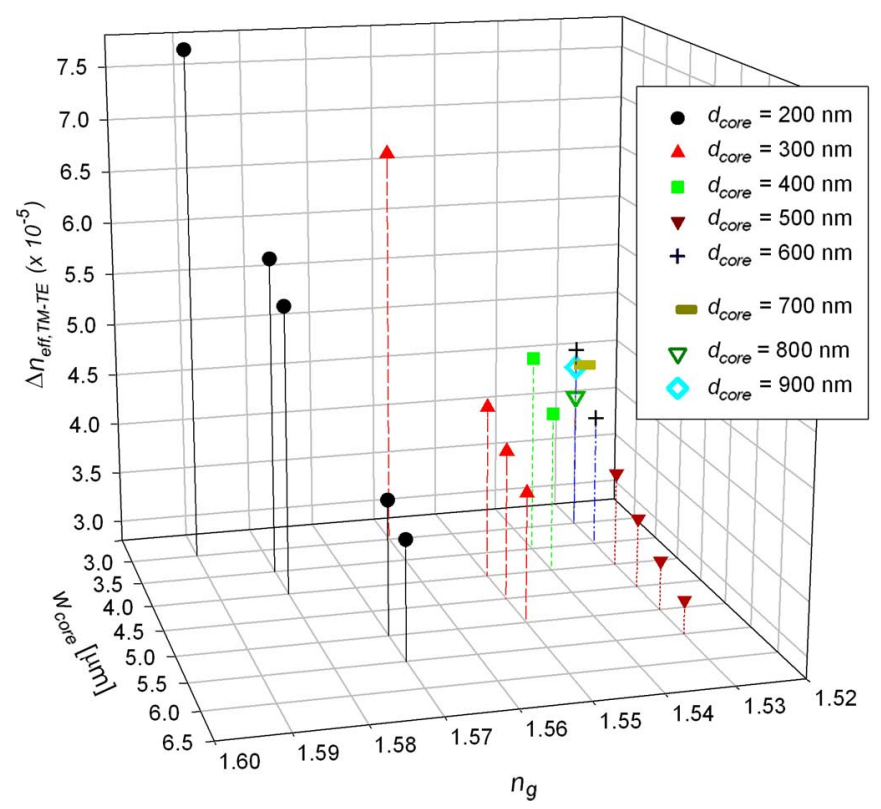

Fig. 3. Worst-case values for technological tolerance impact on the channel birefringence of various PIWs given by sets of $\left[w, n_{g}, d_{\text {core }}\right]$ at a core-layer materials birefringence of $2 \times 10^{-3}$.

in the WGM regime. Then, the impact of all the technological tolerances on the channel birefringence has been analyzed for all PIW structures, fulfilling the aforementioned criteria. The maximum impact of the uncertainty $\Delta x$ in each technological parameter $x$, on the polarization dependence, defined by $\left|\left(\partial \Delta N_{\mathrm{TE}-\mathrm{TM}} / \partial x\right) \Delta x\right|$, is given in Table III. Furthermore, trends in the changes of the local impact values over the entire $n_{g}, d_{\text {core }}$, and $w_{\text {core }}$ range are presented.

By adding the absolute impact values of the technological tolerances of the core thickness $\delta d$, core refractive index $\Delta n_{g}$, and channel width $\Delta w$, a worst-case value of the total impact of all technological uncertainties on the polarization dependence can be calculated. For the sets of waveguide parameters, selected by the previously described criteria, these total impacts are presented in Fig. 3. Here, a fixed value of the materials birefringence of the core layer $\left(2 \times 10^{-3}\right)$ is assumed. Based on this 3-D plot and on the data presented in Table II, we can easily identify the parameter space with sufficiently low sensitivity of the channel birefringence to technological variations. The requirement of overlap of the ranges given in Table II defines 
the suited range of waveguide parameters, which are $n_{g}$ [1.53-1.54], $d_{\text {core }}[600-900 \mathrm{~nm}]$, and $w_{\text {core }}[3-3.5 \mu \mathrm{m}]$.

In the final design, our polarization-independent channel waveguide [30], based on silicon-oxide/SiON technology, is chosen from that range. The waveguide has a core thickness of $820 \mathrm{~nm}$, a channel width of $3.25 \mu \mathrm{m}$, and a core-to-background index contrast of $6.9 \times 10^{-2}$ for TE-polarized light. The material birefringence of cladding and core layer has been determined to be $1 \times 10^{-3}$ and $2 \times 10^{-3}$, respectively. The effective refractive-index contrast of this waveguide is $1.4 \times 10^{-2}$. This waveguide design is polarization independent, with a worstcase change due to technological tolerances of $4.1 \times 10^{-5}$.

Finally, we want to mention a special feature of the sensitivity of the channel birefringence to variations of the core-layer thickness. This feature holds for a select parameter range of $n_{g}$ [1.51-1.54] and $d_{\text {core }}$ [600-1200 nm] only. In that range, solutions for PIWs can be found for which the change in channel birefringence becomes independent of the layer thickness $\left(\partial \Delta N_{\mathrm{TM}-\mathrm{TE}} / \partial d=0\right)$. With an appropriate choice for the channel width, waveguides were found to fulfill the channel-birefringence-sensitivity criterion $\left(\leq 5 \times 10^{-5}\right)$ over a thickness range of more than $200 \mathrm{~nm}$. This feature, which might become interesting in case processes with considerably worse thickness control are applied, is described in more detail elsewhere [30].

Based on the parameters of our final waveguide channel $\left(d_{\text {core }}=820 \mathrm{~nm}, w_{\text {core }}=3.25 \mu \mathrm{m}\right.$, and $\left.\Delta N=6.9 \times 10^{-2}\right)$, an optimum bend layout and the fiber-to-chip coupling have to be designed. Detail will be presented in the next sections.

\section{B. Design of Waveguide Bends}

The layout of bends in the designed channel waveguide has been optimized in order to minimize the bending radius while keeping the loss below $0.01 \mathrm{~dB} / 90^{\circ}$. A radius $R_{b}<1 \mathrm{~mm}$ has been obtained. The only remaining degree of freedom for this design is the lateral structure of the bent section.

Two types of functional loss can be distinguished in a bentwaveguide section that is connected to two straight waveguides: 1) intrinsic radiation loss of the leaky bend mode, which is called pure bend loss, and 2) transition loss due to modal mismatch between light propagating through the straight- and bent-waveguide sections.

Bent-waveguide sections are often chosen to have a channel width identical to that of the straight waveguides. Transition loss can be reduced by offsetting the channel centers at the transitions. Also, a uniform bending radius is often chosen, with a value large enough to satisfy the requirements to the total bending loss. In our approach, the bends have a positiondependent bending radius and width, and the discontinuous transition is replaced by a gradual adiabatic one. In addition, the waveguide width in the main part of the bend is chosen large enough to allow for a WGM, which reduces pure bend loss. The resulting adiabatic bend is more compact than traditionally designed ones for a given maximum total bend loss.

In a first design step, the pure bend loss has been calculated as a function of the bending radius and channel width, both being constant over the bend, applying a finite-difference 2-D

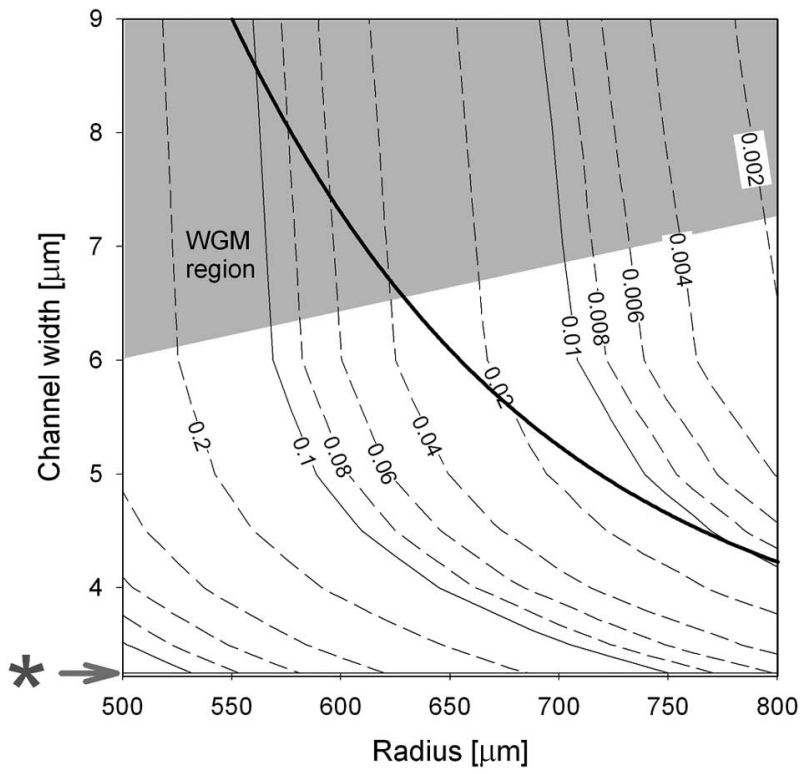

Fig. 4. Contour plots of the pure bend loss as function of radius and waveguide width $\left(^{*}=\right.$ waveguide width of straight channel, $\left.3.25 \mu \mathrm{m}\right)$. The values at the contour lines indicate the loss in decibels per $180^{\circ}$. The heavy solid line shows the chosen taper function of waveguide width versus local radius.

TABLE IV

COMPARISON OF LOSS AND SIZE OF $180^{\circ}$ BENDS OF Two TyPES OF Conventional (Conv.) Bend Designs and the Adiabatic Bend DESCRIBED IN THIS SECTION

\begin{tabular}{|c|c|c|c|}
\hline Bend type & Conv. I & Conv. II & Adiabatic \\
\hline Offset & None & Yes & None \\
\hline Bend radius $[\mu \mathrm{m}]$ & 652 & 652 & Var. $\geq 600$ \\
\hline Bend width $[\mu \mathrm{m}]$ & 3.25 & 3.25 & Var. $3.25-7.3$ \\
\hline Transition loss $[\mathrm{dB}]$ & 0.097 & 0.034 & $<0.005$ \\
\hline Pure bend loss $[\mathrm{dB}]$ & 0.28 & 0.28 & 0.029 \\
\hline Total loss $[\mathrm{dB}]$ & 0.474 & 0.348 & 0.029 \\
\hline Size $\left[\mathrm{mm}^{2}\right]$ & 0.85 & 0.85 & 0.85 \\
\hline
\end{tabular}

bend-mode-calculation method [27]. Results are shown in the contour plots of Fig. 4. The data clearly shows that the pure bend loss, given by the contour-value labels, decreases with increasing bend radius and channel width. From our calculations, we conclude that, in this bend configuration of our designed waveguide $(w=3.25 \mu \mathrm{m})$, pure bend loss $<0.02 \mathrm{~dB} / 90^{\circ}$ can be obtained for bending radii down to $1 \mathrm{~mm}$.

Based on this general calculation, a bend with adiabatically changing channel width and radius has been designed in order to minimize the transition loss as well. This optimization involves a tradeoff between pure bend loss, transition loss, and needed chip area. The precise bend structure, specifying both the bend radius and the waveguide width as a function of position in the bend, has been presented in [31]. The chosen adiabatic width taper function versus local bend radius is shown in Fig. 4 (heavy solid line).

In Table IV, the loss of our adiabatic bend is compared to that of two different conventionally designed bends (one without offset, the other with optimized offset), where in all cases, the bend radius has been chosen such that the bends occupy identical chip areas. The adiabatic design clearly shows a strong loss reduction. 


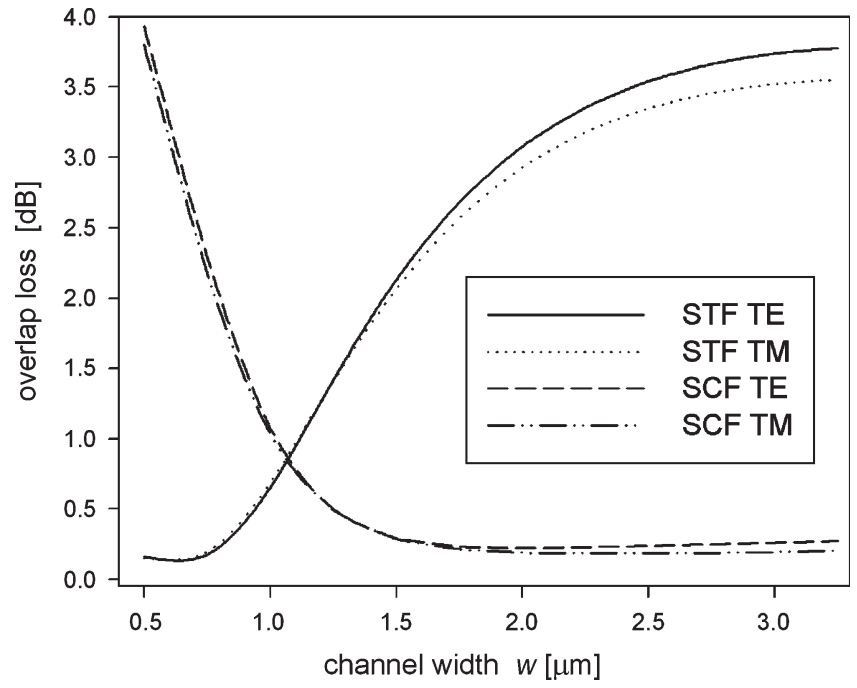

Fig. 5. Overlap loss between channel waveguide and optical fiber (STF: standard $9-\mu \mathrm{m}$ telecom fiber, SCF: $4-\mu \mathrm{m}$ high numerical aperture small-core fiber) versus channel width.

We checked, by numerical calculations, that the bendinduced birefringence of the standard width waveguide is smaller than the straight waveguide birefringence for all bend radii down to $600 \mathrm{~nm}$, but the wider waveguide sections needed in the adiabatically tapered bend design show larger birefringence as the radius becomes less than $1 \mathrm{~mm}$. For Mach-Zehnder-type wavelength filters, this bend-induced birefringence is unimportant because the bends in both branches can be made equal, thus canceling out this birefringence term. However, for ring-resonator devices, no such cancellation is possible, and the ring radius should be kept larger than $1 \mathrm{~mm}$.

\section{Fiber-to-Chip Coupling}

For the coupling of light from standard optical fibers into the waveguides of the optical chips, high coupling efficiency ( $<1-\mathrm{dB}$ loss per facet), minimized polarization-dependent coupling loss (PDCL), and reduced sensitivity to fiber misalignment are of major importance. As aforementioned, the relatively high lateral index contrast in our channel design results in a field profile which has a poor overlap with the optical field in the standard fiber. Consequently, a fiber-to-chip loss of $\alpha_{\mathrm{fc}}=3.7 \mathrm{~dB} /$ facet and a PDCL of $0.23 \mathrm{~dB}$ would be obtained (see Fig. 5). It is well known that below a certain channel width, further width reduction will result in an increased size of the modal field, enabling matching to the initially larger modal field in the optical fiber [12]. This approach is based on channel-size reduction by adiabatic tapering of its width only. The reduction of overlap loss, which is obtained by using this principle, can be deduced from Fig. 5. Around a channel width of $0.75 \mu \mathrm{m}$, the overlap loss and PDCL are minimized to $0.19 \mathrm{~dB} /$ facet and $0.02 \mathrm{~dB}$, respectively. Fabrication of waveguide tapers with such a small final channel width, however, will cause serious problems due to limited resolution of the applied standard contact-lithographic and etching processes.

Therefore, we have chosen another approach in which the mode field diameter of the fiber is significantly reduced by splicing the standard telecom fiber to a high numerical aperture

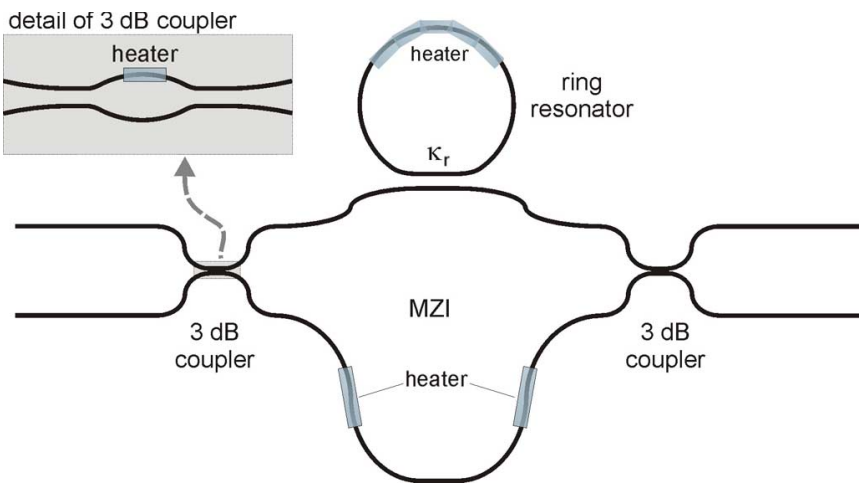

Fig. 6. Schematic layout of optical wavelength filter with detail of a 3-dB coupling section shown in the inset [33].

small-core fiber with a 4- $\mu \mathrm{m}$ mode field diameter. Since the optimized fiber splice results in a nearly adiabatic spot-size converter, this additional step only adds about $0.2 \mathrm{~dB} /$ splice to the overall coupling loss [32]. This approach accomplishes an increase of the optimized channel width to $2 \mu \mathrm{m}$, which considerably relaxes the requirements to the fabrication processes. At this channel width, the fiber-to-chip coupling loss from the $4-\mu \mathrm{m}$ fiber will be below $0.25 \mathrm{~dB}$ per facet with a PDCL of about $0.03 \mathrm{~dB}$. The alignment tolerance is better than $0.5 \mathrm{~dB}$ for a misalignment up to $0.4 \mu \mathrm{m}$, which is a sufficiently safe margin for fiber-array alignment. A calculation of the total coupling loss shows that our designed waveguide amply fulfills the demand of $1 \mathrm{~dB} /$ facet coupling loss.

\section{Test-Structure Design}

A sensitive test structure is needed to evaluate the small residual birefringence of the waveguides. For this, we have used a passsband-flattened optical wavelength interleaver filter, which was also investigated, in our group [32]. This filter shows a steep-sloped transfer function which facilitates accurate measurement of the spectral TE-TM shift that is caused by channel birefringence. The structure, which is shown schematically in Fig. 6, acts as an interleaver with an FSR of $50 \mathrm{GHz}$ $(\sim 0.4 \mathrm{~nm})$, and it can be thermo-optically tuned over its full FSR. The filtering principle is based on a Mach-Zehnder interferometer (MZI) with two 3-dB couplers and branches with unequal length in-between. A ring resonator is connected the shorter branch, which introduces a phase shift that varies periodically with frequency in a nonlinear way. If the wavelength periodicity of the ring response is half the periodicity (FSR) of the MZI, the ring-induced phase shift may compensate the linear phase shift of the longer MZI branch over a significant part of the FSR. This leads to a steep-sloped transfer function with a flattened passband and a broadened stopband. The $3-\mathrm{dB}$ couplers of the filter device are tunable MZI couplers with a heater placed on one of the branches (see inset of Fig. 6), enabling the attainment of an exact 3-dB splitting ratio, even in the presence of fabrication inaccuracies.

For the tuning of the filter characteristics, heaters of $1.5 \mathrm{~mm}$ length have been placed on the ring and on the long branch of the MZI. The design of the ring and its coupling section with the MZI branch aimed at maximum passband flattening, leading 
TABLE V

FSR $\left(\Delta f_{\mathrm{FSR}}\right.$ AND $\left.\Delta \lambda_{\mathrm{FSR}}\right)$, MZI BRANCH-LENGTH DIFFERENCE $\left(\Delta L_{\text {MZI }}\right)$, Ring Path Length $\left(L_{\text {Ring }}\right)$, AND the Minimum Radius of AdiABATIC BENDS IN THE Ring $\left(R_{\text {ring,min }}\right)$

\begin{tabular}{|l|l|l|l|l|}
\hline$\Delta f_{F S R}[\mathrm{GHz}]$ & $\Delta \lambda_{F S R}[\mathrm{~nm}]$ & $\Delta L_{M Z I}[\mathrm{~mm}]$ & $L_{\text {Ring }}[\mathrm{mm}]$ & $R_{\text {ring,min }}[\mathrm{mm}]$ \\
\hline 50 & 0.4 & 3.997 & 7.994 & 1.1 \\
\hline
\end{tabular}

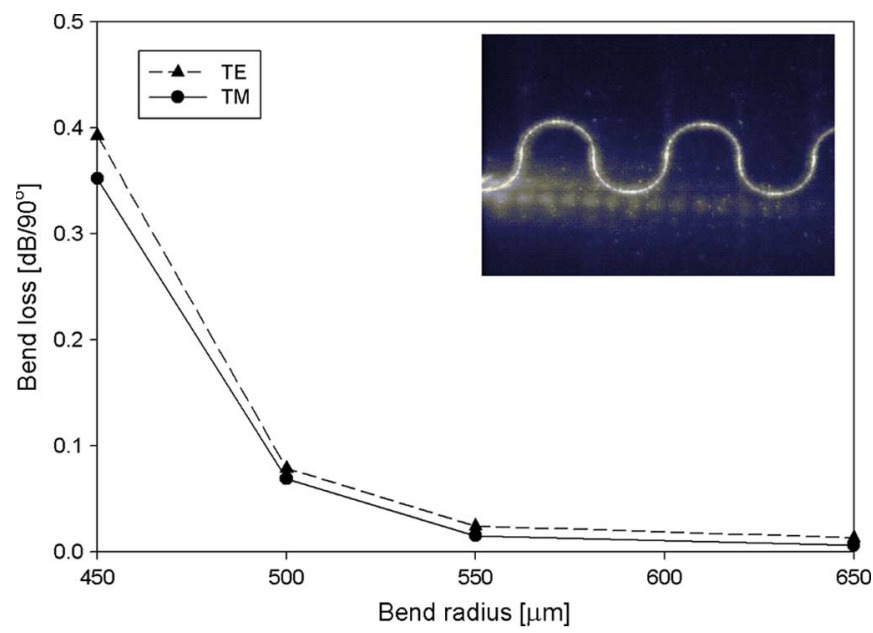

Fig. 7. Bending loss of adiabatic bends as function of the minimum bending radius. The inset shows a photograph of light propagation in a realized bend with $700-\mu \mathrm{m}$ bending radius.

to a calculated optimum power-coupling ratio $\kappa_{r}=0.82$. The filter presented in this paper has been designed for an FSR $\left(\Delta f_{\mathrm{FSR}}\right)$ of $50 \mathrm{GHz}$ and a crosstalk of $-29 \mathrm{~dB}$. The parameters of this filter design are given in Table $\mathrm{V}$.

\section{FAbRication And Results}

The device has been fabricated using the standard siliconoxynitride technology available in our laboratory [12], [27]. The PECVD SiON core layer was deposited on top of an $8-\mu \mathrm{m}$ thermal oxide layer on $\langle 100\rangle$ silicon wafers. The layerthickness and refractive-index values of the as-deposited film were chosen so that after all annealing processes, their values should correspond to those of the waveguide design. A first annealing process $\left(1 \mathrm{~h}\right.$ at $1150^{\circ} \mathrm{C}$ ) has been applied in order to remove the loss-inducing hydrogen bonds. Then, the waveguide channels have been defined by applying standard lithography and reactive-ion etching. The waveguide channels have been overgrown by a 7- $\mu \mathrm{m}$ PECVD silicon-oxide cladding layer, followed by a second annealing process, in order to tune the refractive index (1.463, as-deposited) of this top cladding layer to the refractive-index value of the thermal oxide (1.450). Finally, the heater elements and electrical leads have been applied by lift-off technique in combination with chromium and aluminum deposition, respectively.

For testing the adiabatic-bend design, waveguiding channels, which have been configured as a meandering cascade of 52 bends of $90^{\circ}$ each, have been designed. The minimum bend radius was varied from 450 to $700 \mu \mathrm{m}$. The inset of Fig. 7 shows light propagation (as evidenced by residual scattered light) through a part of such a cascade with bends having a minimum radius of $700 \mu \mathrm{m}$. The experimental results given in the main part of Fig. 7 clearly show that the loss criterion

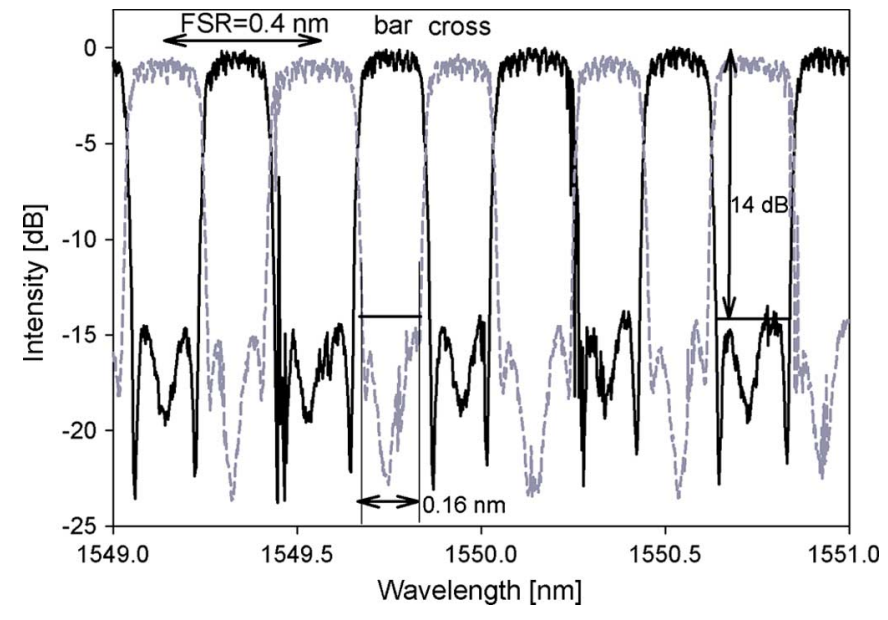

Fig. 8. Measured normalized TM response at both outputs of the filter structure: bar (black) and cross (gray).

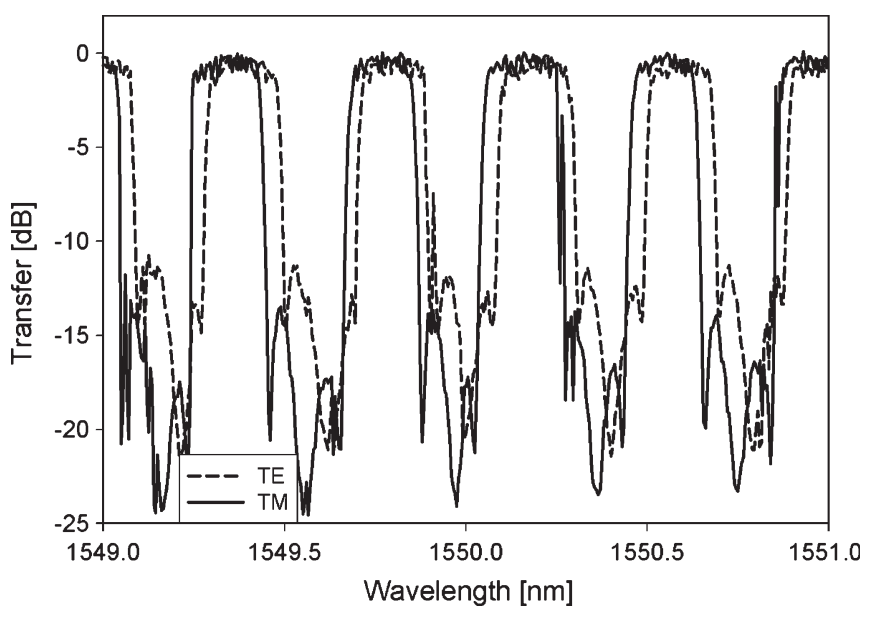

Fig. 9. Measured normalized bar response for TM- and TE-polarized light.

$\left(\alpha<0.02 \mathrm{~dB} / 90^{\circ}\right)$ is fulfilled at a bending radius as low as $600 \mu \mathrm{m}$. The polarization dependence of the bending loss was measured to be below $8 \times 10^{-3} \mathrm{~dB}$ for all minimum bend radii $>500 \mu \mathrm{m}$.

The measured filter response of our test structure (the wavelength interleaver) applying TM-polarized light is shown in Fig. 8. Passband-flattened and stopband-broadened response curves with steep slopes are clearly observed. The measured FSR of $50 \mathrm{GHz}$ exactly corresponds to the designed value. The measured crosstalk of $-14 \mathrm{~dB}$, however, is larger than the crosstalk aimed at by the design. The increased crosstalk can easily be explained by the deviation of the power-coupling coefficient $\kappa_{r}$ to the ring ( 0.59 was measured in stead of the designed value of 0.82 ). Recalculation of the filter response, inserting the measured value $\kappa_{r}=0.59$, resulted in excellent agreement between the theoretically calculated and experimentally obtained response of the add-drop filter. The residual channel birefringence was derived from the observed TE-TM shift of the filter response. A measurement with TE-polarized light showed that both the ring and the main MZI opticalpath-length difference were slightly detuned. Fig. 9 shows both the TM and the TE response of the test device after retuning only the ring in order to restore the passband-flattened shape 
of the transfer function but without retuning the MZI. Since the tuning of the filter is determined only by the optical-pathlength difference of the MZI branches, the birefringence of these branches can be calculated from the measured TE-TM shift. Although the measurements shown in Fig. 8 show some uncertainty in the wavelength scale due to mode hopping of the source laser, an average $0.03-\mathrm{nm}$ TE-TM shift can be determined, corresponding to a channel birefringence of $3 \times$ $10^{-5}$. The possibility of a birefringence shift over multiple FSRs can be excluded, because in several devices designed to have different FSR values, exactly the same TE-TM shift has been observed. From this, we conclude that our objective of designing and fabricating highly tolerant waveguide channels with a birefringence below $5 \times 10^{-5}$ has been realized. Despite the TE/TM shift, this $25-\mathrm{GHz}$ channel-spacing device satisfies the Telcordia GR-1209 specifications for the passband shape.

\section{CONCLUSION}

In this paper, we have presented a tolerance-analysis-based design of a nearly polarization-independent optical waveguide structure, which fulfills the strong demands arising from the field of communications application: low loss, high compactness (small bending radius, $<1 \mathrm{~mm}$ ), efficient (loss $<1 \mathrm{~dB}$ per facet), and polarization-independent fiber-to-chip coupling; and very low-polarization dependence $\left(\Delta N_{\mathrm{TE}-\mathrm{TM}}<5 \times 10^{-5}\right)$. This design was evaluated by using a passband-flattened wavelength interleaver filter having a 50-GHz FSR as a test device. This filter, which is based on a Mach-Zehnder configuration in combination with a ring resonator, which clearly showed the designed passband flattening of the response, enabled us to accurately measure the TE-TM shift of $0.03 \mathrm{~nm}$, from which the value of the channel birefringence $3 \times 10^{-5}$ was determined.

\section{ACKNOWLEDGMENT}

The authors would like thank G. Sengo, A. Hollink, and L. Hilderink for their technical support and their students A. Leinse and R. Harbers.

\section{REFERENCES}

[1] R. C. Alferness, H. Kogelnik, and T. H. Wood, "Semiconductors and photonics-The evolution of optical systems: Optics everywhere," Bell Labs Tech. J., vol. 5, no. 1, pp. 188-202, Jan./Mar. 2000.

[2] L. Eldada, "Optical communication components," Rev. Sci. Instrum., vol. 72, no. 3, pp. 575-593, Mar. 2004.

[3] M. R. Poulsen, P. I. Borel, J. Fage-Pedersen, J. Hübner, M. Kristensen, J. H. Poulsen, K. Rottwitt, M. Svalgaard, and W. Svendsen, "Advances in silica-based integrated optics," Opt. Eng., vol. 42, no. 10, pp. 2821-2834, 2003.

[4] G. L. Bona, W. E. Denzel, B. J. Offrein, R. Germann, H. W. M. Salemink, and F. Horst, "Wavelength division multiplexed add/drop ring technology in corporate backbone networks," Opt. Eng., vol. 37, no. 12, pp. 3218-3228, Dec. 1998.

[5] T. Miya, "Silica-based planar lightwave circuits: Passive and thermally active devices," IEEE J. Sel. Topics Quantum Electron., vol. 6, no. 1, pp. 38-45, Jan./Feb. 2000.

[6] B. J. Offrein, R. Germann, F. Horst, H. W. M. Salemink, R. Beyeler, and G. L. Bona, "Resonant coupler-based tunable add-after-drop filter in silicon-oxynitride technology for WDM networks," IEEE J. Sel. Topics Quantum Electron., vol. 5, no. 5, pp. 1400-1406, Sep./Oct. 1999.

[7] B. E. Little, S. T. Chu, P. P. Absil, J. V. Hryniewicz, F. G. Johnson, F. Seiferth, D. Gill, V. Van, and O. King, "Passive components-Very high-order microring resonator filters for WDM applications," IEEE Photon. Technol. Lett., vol. 16, no. 10, pp. 2263-2265, Oct. 2004.

[8] M. T. Hill, H. J. S. Dorren, T. de Vries, X. J. M. Leijtens, J. H. den Besten, B. Smalbrugge, Y. S. Oei, H. Binsma, G. D. Khoe, and M. K. Smit, "A fast low-power optical memory based on coupled micro-ring lasers," Nature, vol. 432, no. 7014, pp. 206-209, Nov. 2004.

[9] L. Eldada and L. W. Shacklette, "Advances in polymer integrated optics," IEEE J. Sel. Topics Quantum Electron., vol. 6, no. 1, pp. 54-68, Jan./Feb. 2000.

[10] B. K. Das, R. Ricken, V. Quiring, H. Suche, and W. Sohler, "Distributed feedback-distributed Bragg reflector coupled cavity laser with a Ti : (Fe :)Er : $\mathrm{LiNbO}_{3}$ waveguide," Opt. Lett., vol. 29, no. 2, pp. 165-167, Jan. 2004.

[11] R. Germann, H. W. M. Salemink, R. Beyeler, G. L. Bona, F. Horst, I. Massarek, and B. J. Offrein, "Silicon oxynitride layers for optical waveguide applications," J. Electrochem. Soc., vol. 147, no. 6, pp. 2237-2241, 2000.

[12] K. Wörhoff, P. V. Lambeck, and A. Driessen, "Design, tolerance analysis and fabrication of silicon oxynitride based planar optical waveguides for communication devices," J. Lightw. Technol., vol. 17, no. 8, pp. 1401-1407, Aug. 1999.

[13] C. G. H. Roeloffzen, "Passband flattened binary-tree structured adddrop multiplexers using SiON waveguide technology," Ph.D. dissertation, Univ. Twente, Enschede, The Netherlands, 2002.

[14] D. J. W. Klunder, E. Krioukov, F. S. Tan, T. van der Veen, H. F. Bulthuis, G. Sengo, C. Otto, H. J. W. M. Hoekstra, and A. Driessen, "Vertically and laterally waveguide-coupled cylindrical microresonators in $\mathrm{Si}_{3} \mathrm{~N}_{4}$ on $\mathrm{SiO}_{2}$ technology," Appl. Phys. B, Photophys. Laser Chem., vol. 73, no. 5/6, pp. 603-608, 2001

[15] O. Zywitzki, T. Modes, H. Sahm, P. Frach, K. Goedicke, and D. Glöß, "Structure and properties of crystalline titanium oxide layers deposited by reactive pulse magnetron sputtering," Surf. Coat. Technol., vol. 180-181, no. 1, pp. 538-543, 2004.

[16] T. Tsuchizawa, K. Yamada, H. Fukuda, T. Watanabe, J. Takahashi, M. Takahashi, T. Shoji, E. Tamechika, S. Itabashi, and H. Morita, "Microphotonics devices based on silicon microfabrication technology," IEEE J. Sel. Topics Quantum Electron., vol. 11, no. 1, pp. 232-240, Jan./Feb. 2005.

[17] B. Schüppert, J. Schmidtchen, A. Splett, U. Fischer, T. Zinke, R. Moosburger, and K. Petermann, "Integrated optics in silicon and SiGeheterostructures," J. Lightw. Technol., vol. 14, no. 10, pp. 2311-2323, Oct. 1996.

[18] H. Takahashi, Y. Hibino, Y. Ohmori, and M. Kawachi, "Polarization insensitive arrayed waveguide wavelength multiplexer with birefringence compensating film," IEEE Photon. Technol. Lett., vol. 5, no. 6, pp. 707-709, Jun. 1993.

[19] M. Okuno, A. Sugita, K. Jinguji, and M. Kawachi, "Birefringence control of silica waveguides on $\mathrm{Si}$ and its application to a polarization beam splitter/switch," J. Lightw. Technol., vol. 12, no. 4, pp. 625-633, Apr. 1994.

[20] M. Hoffman, P. Kopka, and E. Voges, "Low-loss fiber-matched lowtemperature PECVD waveguides with small core dimensions for optical communication systems," IEEE Photon. Technol. Lett., vol. 9, no. 9, pp. 1238-1240, Sep. 1997.

[21] H. H. Yaffe, C. H. Henry, R. F. Kazarinov, and M. A. Milbrodt, "Polarization independent silica-on-silicon Mach-Zehnder interferometers," J. Lightw. Technol., vol. 12, no. 1, pp. 64-67, Jan. 1994.

[22] K. Wörhoff, B. J. Offrein, P. V. Lambeck, G. L. Bona, and A. Driessen, "Birefringence compensation applying double-core waveguiding structures," IEEE Photon. Technol. Lett., vol. 11, no. 2, pp. 206-208, Feb. 1999.

[23] J. J. G. M. van der Tol, F. Hakimzadeh, J. W. Pedersen, D. Li, and H. van Brug, "A new short and low-loss passive polarization converter on InP," IEEE Photon. Technol. Lett., vol. 7, no. 1, pp. 32-34, Jan. 1995.

[24] G. L. Bona, R. Germann, and B. J. Offrein, "SiON high-refractive-index waveguide and planar lightwave circuits," IBM J. Res. Develop., vol. 47, no. 2/3, pp. 243-249, Mar. 2003.

[25] B. E. Little, "A VLSI photonics platform," in Proc. Opt. Fiber Comm. Conf., 2003, vol. 2, pp. 444-445.

[26] M. G. Hussein, K. Wörhoff, G. Sengo, and A. Driessen, "Deposition and characterization of PECVD phosphorus-doped silicon oxynitride layers for integrated optics applications," in Proc. EURO CVD, 2005, pp. 457-464.

[27] K. Wörhoff, A. Driessen, P. V. Lambeck, L. T. H. Hilderink, P. W. C. Linders, and T. J. A. Popma, "Plasma enhanced chemical vapor deposition of silicon oxynitride optimized for application in integrated optics," Sens. Actuators A, Phys., vol. 74, no. 1, pp. 9-12, Apr. 1999. 
[28] TempSelene, BBV Software BV. version 4.1.00.

[29] M. K. Smit, E. C. M. Pennings, and H. Blok, "A normalized approach to the design of low-loss optical waveguide bends," J. Lightw. Technol., vol. 11, no. 11, pp. 1737-1742, Nov. 1993.

[30] K. Wörhoff, "Polarisatieonafhankelijke lichtgeleidende structuur," Dutch Patent NL 1019 488, Feb. 12, 2002.

[31] C. G. H. Roeloffzen, R. M. de Ridder, and A. Driessen, "Low-loss adiabatic bend using minimized chip area," in Proc. IEEE LEOS Symp. Benelux Chapter, 2000, pp. 175-178.

[32] A. Leinse, C. G. H. Roeloffzen, K. Wörhoff, G. Sengo, R. M. de Ridder, and A. Driessen, "Low-loss fiber to chip connection system for telecommunication devices," in Proc. IEEE LEOS Symp. Benelux Chapter, 2001, pp. $185-188$.

[33] C. G. H. Roeloffzen, R. M. de Ridder, G. Sengo, K. Wörhoff, and A. Driessen, "Passband flattening and rejection band broadening of a periodic Mach-Zehnder wavelength filter by adding a tuned ring resonator," in Proc. ECOC, 2002, vol. 3, p. 1.20.

Kerstin Wörhoff received the M.Sc. degree in optoelectronics from the Technical University of Bratislava, Bratislava, Slovak Republic, in 1991 and the Ph.D. degree in applied physics from the University of Twente, Enschede, The Netherlands, in 1996.

In 1996, she joined the Lightwave Devices Group (now Integrated Optical MicroSystems Group, MESA+ Institute for Nanotechnology), University of Twente, as a Postdoctoral Researcher. Since 2000, she has been an Assistant Professor with the same group. Her research interest covers the field of siliconbased photonics technology and integrated optical waveguide design. She has (co-)authored more than 50 journal and conference papers and one patent.

Chris G. H. Roeloffzen (S'98-M'03) was born in Almelo, The Netherlands, in 1973. He received the M.Sc. degree in applied physics and the Ph.D. degree in electrical engineering from the University of Twente, Enschede, The Netherlands, in 1998 and 2002, respectively.

From 1998 to 2002, he was with the Integrated Optical MicroSystems Group, MESA+ Institute for Nanotechnology, University of Twente, where he was involved in research on integrated optical add-drop demultiplexers in silicon oxynitride waveguide technology. In 2002, he became an Assistant Professor in the Telecommunication Engineering Group, University of Twente. He is now involved with research and education on optical fiber communications systems. His current research interests include optical communications and RF photonic signal processing techniques.
René M. de Ridder (M'94) was born in Amsterdam, The Netherlands, in 1950. $\mathrm{He}$ received the M.S. and Ph.D. degrees in electrical engineering from the University of Twente, Enschede, The Netherlands, in 1978 and 1988, respectively. His thesis subject was on sensitive thin-film magnetic-field sensors.

Since 1981, he has been a Lecturer and a Research Scientist at the University of Twente, where he is currently a member of the Integrated Optical MicroSystems Group, MESA+ Institute for Nanotechnology. In 1988, his research focus turned to integrated optical devices. A basis for his work in this field was laid during a half year of sabbatical leave at AT\&T Bell Labs, Holmdel, NJ, in 1989. His current research interests include photonic crystal structures and wavelength-selective devices for applications in sensing and communication.

Alfred Driessen (M'92-SM'96) received the M.Sc. degree in experimental physics and the Ph.D. degree in quantum solids from the University of Amsterdam, Amsterdam, The Netherlands, in 1972 and 1982, respectively.

After a period as a Postdoctoral Researcher at the Free University of Amsterdam, Amsterdam, where he studied metal hydrides, in 1988, he joined the Lightwave Devices Group (now Integrated Optical MicroSystems (IOMS) Group, MESA+ Institute for Nanotechnology), University of Twente, Enschede, The Netherlands, as an Associate Professor. Since then, he has worked on integrated optics for optical communication and optical nonlinear devices. Since 2003, he has been a Full Professor, and his interest is increasingly focused on compact and complex (nano-) photonic structures, like microresonators and photonic wires, that can be applied in sensing, communication, and spectroscopy. Within IOMS, he is responsible for the Photonics Integration Technology subgroup. He has (co-)authored about 200 refereed journal and conference papers and three patents.

Paul V. Lambeck was born in Amstelveen, The Netherlands, in 1939. He received the M.Sc. degree in physical chemistry from the University of Amsterdam, Amsterdam, The Netherlands, and the Ph.D. degree from the University of Twente, Enschede, The Netherlands, with a thesis on the domain structure stabilization in manganese-doped barium titanate monocrystals.

Since 1964, he has been with the University of Twente, first as an Assistant Professor, and since 1975, as an Associate Professor, and since January 2003 , as a Full Professor of Integrated Optical Sensors. Since March 1, 2004, he formally retired, but he has been with the Integrated Optical MicroSystems Group, MESA+ Institute for Nanotechnology, University of Twente, where he has been continuing his universitary activities in the framework of a "zeroemployment." In 2004, he also joined the Dutch SME OptiSense as the Chief Technology Officer. During the period of 1964-1984, his research was in the area of ferroelectric materials. Since 1984, his research has been in the field of integrated optical devices and microsystems for telecommunication and sensing. He has (co-)authored about 90 scientific publications. He is also the holder of several patents. 